\title{
Superradiant Raman scattering in an ultracold Bose gas at finite temperature
}

\author{
H. Uys* and P. Meystre \\ Department of Physics and B2 Institute, The University of Arizona, Tucson, AZ, 85721
}

\begin{abstract}
We study superradiant Raman scattering from an ultra-cold, but finite temperature Bose gas in a harmonic trap. Numerical simulations indicate the existence of distinct timescales associated with the decoherence of the condensed versus thermal fractions, and the concomitant preferred scattering from atoms in low lying trap states in the regime where superradiance takes place on a timescale comparable to an inverse trap frequency. As a consequence the scattered atoms experience a modest reduction in temperature as compared to the unscattered atoms.
\end{abstract}

\section{INTRODUCTION}

Bose-Einstein condensation (BEC) is characterized by the appearance off-diagonal long-range order, with phase correlations between points spatially separated over macroscopic distances. These correlations are quantified by the first-order spatial correlation function $G^{(1)}\left(\mathbf{x}, \mathbf{x}^{\prime}\right)=\left\langle\hat{\psi}^{\dagger}(\mathbf{x}) \hat{\psi}\left(\mathbf{x}^{\prime}\right)\right\rangle$, where $\hat{\psi}^{\dagger}(\mathbf{x})$ is the field operator that creates a particle at position $\mathbf{x}$. In the thermodynamic limit $G^{(1)}\left(\mathbf{x}, \mathbf{x}^{\prime}\right)$ becomes finite-valued for infinitely separated spatial points below the critical temperature. In ultracold atomic systems the presence of long-range phase correlations is experimentally manifest through the direct observation of matter-wave interference patterns. Hence, matter-wave interference has been used as a diagnostic tool to demonstrate the appearance of the superfluid to Mott-insulator transition [1], the growth of spatial correlations during the formation of a Bose-condensate from a non-equilibrium situation after sudden quenching across the transition point [2], and to measure the critical exponent characterizing the divergence of the correlation length near that point [3].

Another tool recently employed to probe the BEC transition is the selective sensitivity of superradiant scattering to the condensed fraction of an ultra-cold Bose gas beneath the critical point for condensation [4]. Early demonstrations of superradiance were realized in thermal gases at temperatures in the several hundreds of degrees range, $[5,6,6,6,8]$. The majority of these experiments involving initially inverted atomic transitions in relatively high-density samples, a situation often referred to as superfluorescence [8, 9]. By contrast, recent experiments in ultra-cold atomic vapors involved off-resonant light scattering in very low density samples. This regime was first studied in 1999 [10] and has since been the focus of several experiments, such as in the context of coherent matterwave amplification [11, 12, 13]. The majority of these studies used almost pure condensates in which superradiant enhancement is strong due to the near absence of Doppler dephasing. In addition to related theoretical [14] and experimental work on coherent atomic recoil lasing

\footnotetext{
* Currently at the Time and Frequency Division, National Institute of Standards and Technology, Boulder, CO, 80305.
}

[15, 16], to our knowledge only two recent experimental studies considered in detail superradiance from ultracold thermal vapors and its behavior as a function of temperature [4, 17].

One important feature of superradiance from a bosonic atomic vapor cooled below the BEC critical temperature is the existence of two well-separated decay time scales, the fast one attributed to the Doppler dephasing of the thermal fraction of the gas, and the other, slower by roughly an order of magnitude, due to the condensed fraction. These separate time scales were observed by Yoshikawa and co-workers [17] and exploited by Sadler et al. 4] to selectively image the condensed fraction of the vapor over a range of temperatures above and below the critical temperature. In the latter experiment [4] superradiance enhanced absorption of light scattering from a prolate sample of condensed bosonic atoms was monitored in real time during the superradiance process. A main goal of the present paper is to provide a theoretical description of superradiance in ultracold bosonic gases to explain the key temperature dependent aspects of the above-mentioned experiments [4, 17], that enable its use as a probe of coherence in Bose condensates. As such, the present paper is an extension to finite temperatures of our previous study [18] of the spatial inhomogeneity observed in absorption images of the probe light at $T=0$.

The paper is organized as follows. Section II discusses our theoretical model of a non-interacting, ultra-cold atomic gas undergoing Raman transitions in a spherical harmonic trap. Section III presents numerical results demonstrating the existence of two decay times in the superradiant signal. Section IV shows that in that regime where the superradiance time scale becomes comparable to a trap frequency, superradiant scattering occurs predominantly from atoms in the low-lying energy states of the trap, hence it may be used to selectively probe those states. We also remark that superradiance may leave the 'cold' and 'hot' atoms in two different electronic states. Finally, section V is a summary and conclusion.

\section{FINITE TEMPERATURE THEORY}

We consider $N$ non-interacting ultracold atoms in a spherical harmonic trap, Fig. 1(a). The atoms undergo $\Lambda$-type Raman scattering between two electronic ground 
(a)

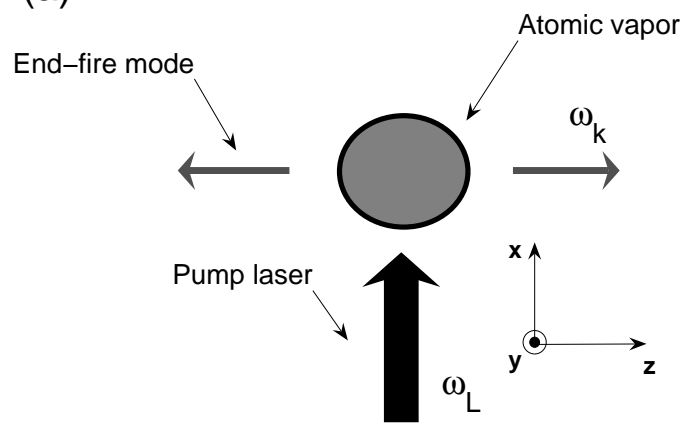

(b)

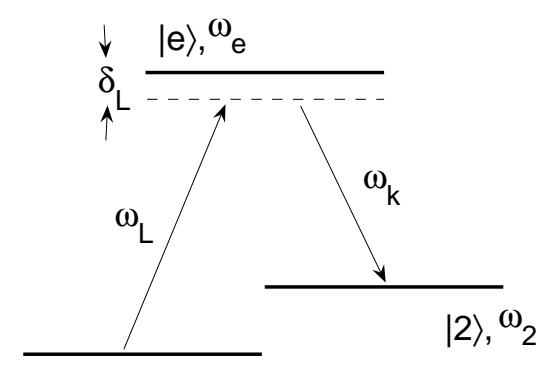

$|1\rangle$

FIG. 1: (a) Experimental setup - a spherically shaped atomic vapor is driven by a laser beam incident along the $x$-axis. The end-fire modes travelling along the $z$-axis are superradiantly amplified. The pump light is assumed to drive a $\Lambda$-type Raman transition for which $\omega_{L} \gg \omega_{2} \gtrsim \delta_{L}$, see Fig. 1 (b)

states $|1\rangle$ and $|2\rangle$ via an excited state $|e\rangle$, see Fig. 1(b). We assume that the transition $|1\rangle \rightarrow|e\rangle$ is driven by an off-resonant classical pump laser $\mathbf{E}_{L}(t)$ of frequency $\omega_{L}$ propagating along the $x$-axis of the trap. For a spherical trap there are no geometrical effects [19] that lead to the selection of particular superradiant modes as would be the case for a cigar-shaped trap, but a preferential direction can be selected by placing the sample in a low finesse ring cavity or by first applying a Bragg pulse to generate a seed matter wave [11, 12]. We assume that this is the case here and that as a result the dominant superradiant modes propagate along the $z$-axis. We refer to them as end-fire modes in analogy to the case of elongated samples. The polarization of the pump light is also chosen parallel to the $z$-axis. For that polarization Rayleigh scattering into the end-fire modes is suppressed due to the angular radiation pattern of Rayleigh scattering. It does however allow Raman scattering into circularly polarized end-fire modes which we describe quantum-mechanically in terms of bosonic creation and annihilation operators $\hat{a}_{\mathbf{k}}^{\dagger}$ and $\hat{a}_{\mathbf{k}}$, respectively. The total electric field is then

$$
\begin{aligned}
\hat{\mathbf{E}} & =\hat{z}\left[E_{L} e^{i\left(\mathbf{k}_{L} \cdot \mathbf{x}-\omega_{L} t\right)}+E_{L}^{*} e^{-i\left(\mathbf{k}_{L} \cdot \mathbf{x}-\omega_{L} t\right)}\right] \\
& +\hat{\epsilon}_{\sigma} \sum_{k}\left[\left(\frac{\hbar \omega_{k}}{2 \epsilon_{0} V}\right)^{\frac{1}{2}} \hat{a}_{\mathbf{k}}(t) e^{i \mathbf{k} \cdot \mathbf{r}}+\text { h.c. }\right],
\end{aligned}
$$

where the incident laser field envelope $E_{L}$ is taken as constant in amplitude. In terms of the detuning

$$
\delta_{L}=\omega_{e}-\omega_{L}
$$

we have

$$
\omega_{k}=\omega_{e}-\omega_{2}-\delta_{L}
$$

We proceed by introducing bosonic matter-field creation and annihilation operators $\hat{\psi}_{i}^{\dagger}(\mathbf{x}, t)$ and $\hat{\psi}_{i}(\mathbf{x}, t)$, that create and annihilate, respectively, an atom at time $t$ and position $\mathbf{x}$ in electronic state $|i\rangle=|1\rangle,|e\rangle$ or $|2\rangle$, with

$$
\left[\hat{\psi}_{i}(\mathbf{x}, t), \hat{\psi}_{j}^{\dagger}\left(\mathbf{x}^{\prime}, t\right)\right]=\delta_{i j} \delta\left(\mathbf{x}-\mathbf{x}^{\prime}\right)
$$

Taking $\omega_{1}=0$ the Hamiltonian of the atom-field system is $\hat{H}=\hat{H}_{0}+\hat{H}_{c}$, with

$$
\begin{aligned}
\hat{H}_{0} & =\sum_{\mathbf{k}} \hbar \omega_{k} \hat{a}_{\mathbf{k}}^{\dagger} \hat{a}_{\mathbf{k}} \\
& +\int d \mathbf{x}\left\{\hbar \omega_{e} \hat{\psi}_{e}^{\dagger}(\mathbf{x}) \hat{\psi}_{e}(\mathbf{x})+\hbar \omega_{2} \hat{\psi}_{2}^{\dagger}(\mathbf{x}) \hat{\psi}_{2}(\mathbf{x})\right\} \\
& +\sum_{i=1,2, e} \int d \mathbf{x} \hat{\psi}_{i}^{\dagger}(\mathbf{x})\left[-\frac{\hbar^{2} \nabla^{2}}{2 m}+V(\mathbf{x})\right] \hat{\psi}_{i}(\mathbf{x}),(4)
\end{aligned}
$$

while

$\hat{H}_{c}=-\int d \mathbf{x}\left\{\hat{\mathbf{E}} \cdot \mathbf{d}\left[\hat{\psi}_{e}^{\dagger}(\mathbf{x}) \hat{\psi}_{1}(\mathbf{x})+\hat{\psi}_{e}^{\dagger}(\mathbf{x}) \hat{\psi}_{2}(\mathbf{x})\right]+\right.$ h.c. $\}$

describes the electric dipole interaction between the atoms and the electromagnetic field, $\mathbf{d}$ being the dipole moment which we take to have the same magnitude for both transitions.

For large enough detunings the excited state is not significantly occupied and may be adiabatically eliminated. Introducing slowly varying operators $\tilde{\psi}_{i}=\hat{\psi}_{i} e^{i \Omega_{i} t}$ and $\tilde{a}_{\mathbf{k}}=\hat{a}_{\mathbf{k}} e^{i \omega_{\mathbf{k}} t}$ and using the rotating wave approximation yields then the effective interaction Hamiltonian 


$$
H_{\text {eff }}=-\int \frac{\hbar}{\delta_{L}} d \mathbf{x}\left\{\Omega_{L}^{2} \hat{\psi}_{1}^{\dagger}(\mathbf{x}) \hat{\psi}_{1}(\mathbf{x})+\Omega_{k}^{2} \hat{\psi}_{2}^{\dagger}(\mathbf{x}) \hat{\psi}_{2}(\mathbf{x}) \hat{a}_{\mathbf{k}}^{\dagger} \hat{a}_{\mathbf{k}}+\Omega_{L} \Omega_{k}\left(\hat{\psi}_{2}^{\dagger}(\mathbf{x}) \hat{\psi}_{1}(\mathbf{x}) \tilde{a}_{\mathbf{k}} e^{i\left(\mathbf{k}_{L}-\mathbf{k}\right) \cdot \mathbf{x}}+\text { h.c. }\right)\right\}
$$

Here $\Omega_{L}=d E_{L} / \hbar$ is the Rabi frequency of the incident field and $\Omega_{k}=d \sqrt{\omega_{k} /\left(2 \epsilon_{0} \hbar V\right)}$.

Expanding the atomic field operators in states of the trap,

$$
\hat{\psi}_{i}(\mathbf{x})=\sum_{i=0}^{\infty} \phi_{n}(\mathbf{x}) \hat{c}_{n i}
$$

where $H_{0} \phi_{n}(\mathbf{x})=\hbar \nu_{n} \phi_{n}(\mathbf{x})$ and the subscript $n$ labels generically excitations in all three dimensions of the trap, $n=\left\{n_{x}, n_{y}, n_{z}\right\}$, and $\nu_{n}=\left(n_{x}+n_{y}+n_{z}+3 / 2\right) \omega_{t}$, substituting the expansion (7) into Hamiltonians (6) and (4), and denoting the expectation values $\left\langle\tilde{a}_{\mathbf{k}}\right\rangle=a_{\mathbf{k}}$, we obtain the Heisenberg equations of motion

$\frac{\partial\left\langle\tilde{c}_{j 1}^{\dagger} \tilde{c}_{n 1}\right\rangle}{\partial \tau}=i \sum_{\mathbf{k} m}\left[\eta_{n m}(-\mathbf{q}) a_{\mathbf{k}}^{\dagger}\left\langle\tilde{c}_{j 1}^{\dagger} \tilde{c}_{m 2}\right\rangle e^{i\left(\nu_{n}^{\prime}-\nu_{m}^{\prime}\right) \tau}-\eta_{j m}^{*}(-\mathbf{q}) a_{\mathbf{k}}\left\langle\tilde{c}_{m 2}^{\dagger} \tilde{c}_{n 1}\right\rangle e^{i\left(\nu_{m}^{\prime}-\nu_{j}^{\prime}\right) \tau}\right]$
$\frac{\partial\left\langle\tilde{c}_{j 2}^{\dagger} \tilde{c}_{n 2}\right\rangle}{\partial \tau}=i \sum_{\mathbf{k} m}\left[\eta_{n m}(\mathbf{q}) a_{\mathbf{k}}^{\dagger}\left\langle\tilde{c}_{j 2}^{\dagger} \tilde{c}_{m 1}\right\rangle e^{i\left(\nu_{n}^{\prime}-\nu_{m}^{\prime}\right) \tau}-\eta_{j m}^{*}(\mathbf{q}) a_{\mathbf{k}}\left\langle\tilde{c}_{m 1}^{\dagger} \tilde{c}_{n 2}\right\rangle e^{i\left(\nu_{m}^{\prime}-\nu_{j}^{\prime}\right) \tau}\right]$
$\frac{\partial\left\langle\tilde{c}_{j 2}^{\dagger} \tilde{c}_{n 1}\right\rangle}{\partial \tau}=i\left[\left(\frac{\Omega_{L}}{\Omega_{k}}-\sum_{\mathbf{k}} \frac{\Omega_{k}}{\Omega_{L}} a_{\mathbf{k}}^{\dagger} a_{\mathbf{k}}\right)\right]\left\langle\tilde{c}_{j 2}^{\dagger} \tilde{c}_{n 2}\right\rangle+i \sum_{\mathbf{k} m}\left[\eta_{n m}(-\mathbf{q}) a_{\mathbf{k}}^{\dagger}\left\langle\tilde{c}_{j 2}^{\dagger} \tilde{c}_{m 2}\right\rangle e^{i\left(\nu_{n}^{\prime}-\nu_{m}^{\prime}\right) \tau}-\eta_{j m}^{*}(\mathbf{q}) a_{\mathbf{k}}\left\langle\tilde{c}_{m 1}^{\dagger} \tilde{c}_{n 1}\right\rangle e^{i\left(\nu_{m}^{\prime}-\nu_{j}^{\prime}\right) \tau}\right]$.

Here the dimensionless time $\tau=\Omega_{R} t$ where $\Omega_{R}=$ $\Omega_{L} \Omega_{k} / \delta_{L}$ is an effective 'two-photon' Rabi frequency, $\nu_{n}^{\prime}=\nu_{n} / \Omega_{R}$, the slowly varying operators $\tilde{c}_{n i}=\hat{c}_{n i} e^{i \nu_{n}^{\prime} t}$ and the overlap function

$$
\eta_{n j}(\mathbf{q})=\int \phi_{n}^{*}(\mathbf{x}) \phi_{j}(\mathbf{x}) e^{i \mathbf{q} \cdot \mathbf{x}} d \mathbf{x}
$$

where the recoil momentum is $\mathbf{q}=\mathbf{k}_{L}-\mathbf{k}$. A grayscale rendering of the absolute value of $\eta_{n j}(\mathbf{q})$ is plotted for the first 25 trap levels and the trap and light field parameters introduced in section III. Finally, the time evolution of the end-fire modes is given by

$$
\begin{aligned}
\frac{\partial a_{\mathbf{k}}}{\partial \tau} & =i \Omega_{k} / \Omega_{L} \sum_{m}\left\langle\tilde{c}_{m 2}^{\dagger} \tilde{c}_{m 2}\right\rangle a_{\mathbf{k}} \\
& +i \sum_{m n} \eta_{m n}(\mathbf{q})\left\langle\tilde{c}_{m 2}^{\dagger} \tilde{c}_{n 1}\right\rangle e^{i\left(\nu_{m}^{\prime}-\nu_{n}^{\prime}\right) t}-\Gamma a_{\mathbf{k}}
\end{aligned}
$$

where the last term is a phenomenological decay added to account for the escape of the photons from the sample. This term should in principle be accompanied by appropriate quantum noise operators to guarantee that the commutation relations of the operators $a_{\mathbf{k}}$ are preserved at all times. These noise operators are also important in that they trigger the superradiant amplification and determine the field fluctuations in its early stages [20, 21]. This paper concentrates however on the later, classical stages of the process, and we follow the standard procedure of introducing a small classical seed for the initial amplitudes of the end-fire modes, which are then described as classical fields. At that level of approximation the phenomenological decay term in Eq. (12) is appropriate. Note also that by factorizing the expectation values of the optical and matter-wave fields, we have neglected any quantum correlation and entanglement that may build up between the optical and matter-wave fields fields.

The atoms obey initially an equilibrium Bose distribution. We determine the distribution for excitations in the $z$-direction by averaging over the $x$ and $y$-directions,

$$
\left\langle c_{n 1}^{\dagger} c_{n 1}\right\rangle=\frac{1}{\mathcal{N}} \sum_{n_{x}, n_{y}=0}^{\infty} \frac{1}{e^{\hbar \omega_{t}\left(n_{x}+n_{y}+n_{z}\right) / k_{B} T}-1},
$$

where $\mathcal{N}$ is a normalization factor chosen to fix the thermal fraction of atoms, $n_{\mathrm{th}}=\sum_{n=0}^{\infty}\left\langle c_{n 1}^{\dagger} c_{n 1}\right\rangle$, at the value $n_{\mathrm{th}}=N\left(T / T_{c}\right)^{3}$ determined by the temperature of the sample [22], where $T_{c}$ is the critical temperature.

We conclude this section by remarking that in order to keep the computations manageable we have assumed that the light field intensity is uniform within the sample, thereby neglecting spatial effects that have previously been shown to play an important role in the growth of the end-fire modes [18, 23, 24]. The role of these effects at finite temperature remains therefore an open question. 


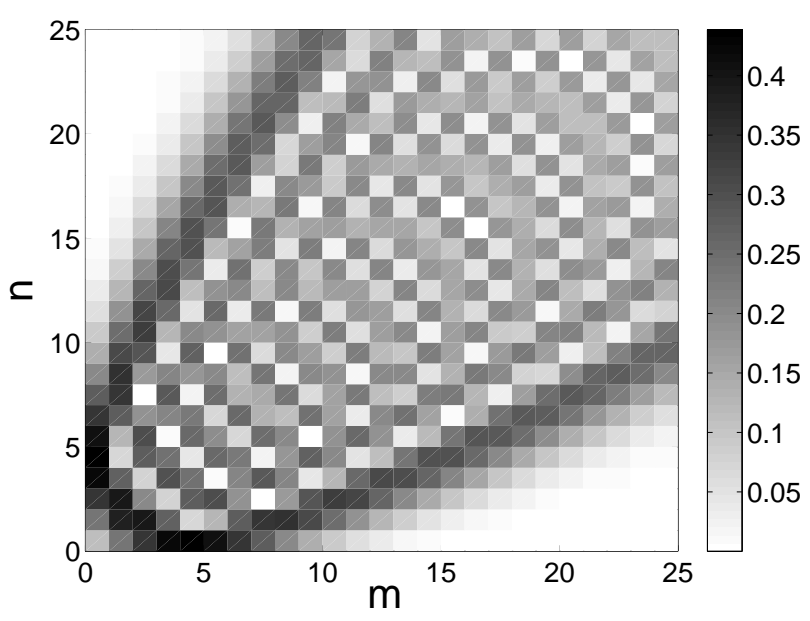

FIG. 2: Gray scale rendering of the absolute value of the overlap function $\left|\eta_{m n}(k)\right|$ for the first 25 trap levels. Here we've assumed a trap frequency $\omega_{t}=2 \pi \cdot 800 \mathrm{rad} / \mathrm{s}$ and $k_{L}=$ $2 \pi / 795 \mathrm{~nm}$ corresponding to the $F=1 \rightarrow F^{\prime}=1$ transition of ${ }^{87} \mathrm{Rb}$.

In the following simulations we also assume for simplicity that the atoms recoil only along the $z$-axis and we are therefore only interested in excitations along that direction.

\section{DEPHASING}

This section summarizes numerical results that illustrate the dynamics of the thermal and condensed fractions of the vapor, showing that these dynamics are characterized by two distinct time scales. Specifically, we simulate a pump-probe technique that was experimentally implemented by Yoshikawa and co-workers [17] to measure superradiant coherence. This procedure entails applying a superradiant pump pulse until the end-fire mode intensity reaches a maximum. The growth of the end-fire mode occurs in response to the build-up of atomic polarization (a polarization grating forms), Eq. (12). At that point the pulse is turned off for a variable delay time, $\tau_{d}$, during which the polarization grating deteriorates due to Doppler dephasing. The pump beam is then turned back on to its original intensity to probe the remaining coherence. Had the polarization grating remained intact the superradiant intensity would immediately return to its pre-delay level, however, due to Doppler dephasing the post-delay intensity is reduced. The ratio of the pre- and post-delay end-fire mode intensities,

$$
\xi=\left|a_{\mathbf{k}}\left(\tau_{\max }+\tau_{d}\right)\right|^{2} /\left|a_{\mathbf{k}}\left(\tau_{\max }\right)\right|^{2},
$$

is therefore a measure of the decoherence of the superradiant pulse.

Figure 3(a) illustrates the detector response (upper curve) to a square pulse pump-probe sequence (lower
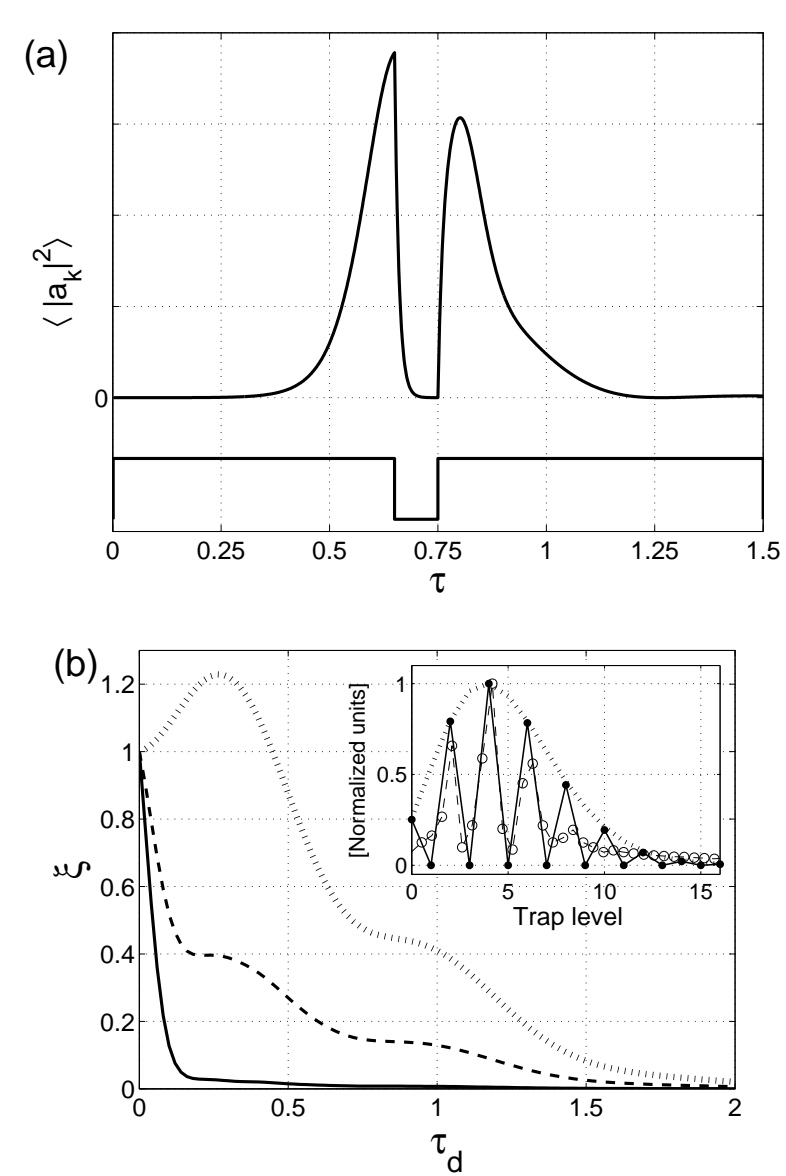

FIG. 3: (a) Pump-probe spectroscopy. The lower curve shows the pump-probe sequence and the upper curve the resulting end-fire mode intensity. The ratio of the peak heights directly after to directly before the delay is used as a measure of coherence. (b) Dephasing. Dotted line: post- to pre-delay intensity signal ratio for a vapor with 0.95 condensed fraction. For a condensed fraction of only 0.1 (solid line) the decay is bimodal, the initial rapid decay being attributed to the thermal fraction. Dashed line: intermediate case, $n_{c}=0.5$. (Inset) Solid circles: polarization $\left|\left\langle\tilde{c}_{m 2}^{\dagger} \tilde{c}_{01}\right\rangle\right|$; open circles Fourier components of the signal for $n_{c}=0.95$ in the main figure. The dotted line envelope shows the overlap function $\left|\eta_{m 0}(\mathbf{q})\right|$.

curve). Here the end-fire mode intensity was convoluted with the response function of the detector, assumed to have a (dimensionless) response time of $\tau^{\prime}=0.01$.

Our simulations are for a sample of $N=10^{6}$ atoms in a three-dimensional, isotropic harmonic trap with trap frequency $\omega_{t}=2 \pi \cdot 800 \mathrm{rad} / \mathrm{s}$ and, with reference to [4] we use the $k_{L}=2 \pi / 795 \mathrm{~nm}$ corresponding to the $F=$ $1 \rightarrow F^{\prime}=1$ transition of ${ }^{87} \mathrm{Rb}$. We use $\omega_{t} / \Omega_{R}=0.5$ and included 400 trap levels in the simulations. A tight trap was chosen for computational reasons: For that value of $\omega_{t}$ the occupation of levels higher than $n=400$ remains negligible at all times for the conditions that we consider 25]. We choose $\Omega_{L} / \Omega_{k}=1000$ and neglect laser pump depletion. Since the decay of the light field due to its 

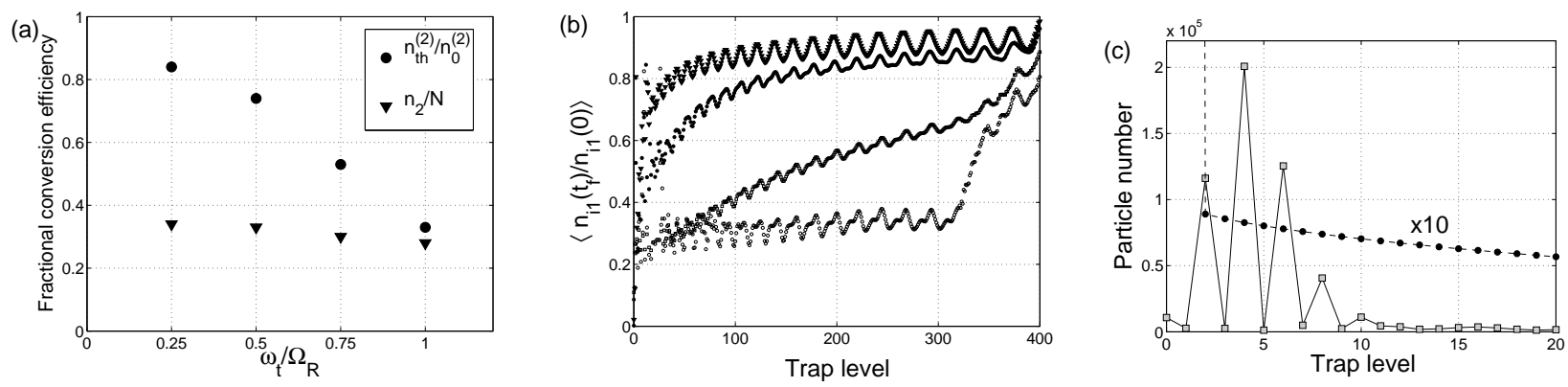

FIG. 4: Summary of numerical simulations for $n_{c}=0.5$. (a) Circles: Ratio of the total number of thermal atoms $\left(n_{\mathrm{th}}^{(2)}\right)$ to total number of condensed atoms $\left(n_{c}^{(2)}\right)$ transferred from the state $|1\rangle$ at peak superradiant intensity ; triangles: fraction of total number of atoms scattered to the state $|2\rangle$ (condensed and thermal included), as a function of $\omega_{t} / \Omega_{R}$. (b) Fraction of atoms left in ground state $|1\rangle$ at the end of the pulse complete by trap level for $\omega_{t} / \Omega_{R}=0.25,0.5,0.75$ and 1.0 . (c) Initial distribution of atoms by trap level (solid circles) and distribution of atoms in state $|2\rangle$ at the end of the pulse for $\omega_{t} / \Omega_{R}=1.0$. The initial distribution was multiplied by a factor of 10 for visibility.

escape from the atomic sample, see Eq. (12), is the fastest process by several orders of magnitude the state of the scattered light field is nearly instantaneously determined by the state of the atomic fields, so that

$$
a_{\mathbf{k}} \approx \frac{i}{\Gamma} \sum_{m n} \eta_{m n}(\mathbf{q})\left\langle\tilde{c}_{m 2}^{\dagger} \tilde{c}_{n 1}\right\rangle e^{i\left(\nu_{m}^{\prime}-\nu_{n}^{\prime}\right) t} .
$$

Either a seed atomic polarization or a seed end-fire mode occupation is required to initiate the superradiant growth. In this paper we usually assume that the end-fire mode has an initial value $a_{\mathbf{k}}=\sqrt{10}$ and that all atoms are in the state $|1\rangle$ obeying the distribution (13). The first numerical iteration of Eqs. (8)-(10) then creates a small initial polarization. Thereafter we use Eq. (15) to determine the instantaneous value of the end-fire mode, with $\Gamma \approx\left(10^{4} \sim 10^{5}\right) \times \Omega_{R}$.

Figure 3(b) plots $\xi\left(\tau_{d}\right)$, the post- to pre-delay intensity ratio, Eq. (14), as a function of the pump-probe delay time for three different sample temperatures. Since the superradiance process is initiated by quantum fluctuations leading to spontaneous emission of photons into the end-fire modes, shot-to-shot fluctuations occur in the relative amplitudes and phases of the left- vs. rightpropagating end-fire modes [18]. To account for these fluctuations we chose, for the purposes of Fig. 3(b), the initial seed amplitudes from a random Gaussian distribution centered around $a_{\mathbf{k}}=\sqrt{10}$ with a standard deviation of $\sigma=\sqrt{10} / 2$ and with a random overall phase. Each curve in Fig. 3(b) represents an average over fifty runs of pump-probe experiments.

The dotted line corresponds to a nearly pure condensate, with a condensed fraction $n_{c}=0.95$. After an initial increase for short times due to the continued build-up of the atomic polarization, the signal ratio decreases to half the initial intensity with a decay time of $\tau_{c} \approx 0.3$.

The slow oscillations in $\xi\left(\tau_{d}\right)$ can be understood by comparing the Fourier transform in one single shot of the experiment to the components of the atomic polarization $\left|\left\langle c_{m 2}^{\dagger} c_{01}\right\rangle\right|$ between the trap ground state and its excited levels, see insert of Fig. 3(b). As expected, there is a oneto-one correspondence between these frequencies. Hence the oscillations in $\xi\left(\tau_{d}\right)$ are a signature of the specific trap characteristics. In the inset the atomic coherence is only significant between even trap states. This is a combined consequence of the two end-fire mode amplitudes and phases having been chosen equal in this shot, and of the form of the overlap function $\left(\eta_{j 0}(q)\right)$ between the lowest trap level and higher levels, which are alternately purely real and purely imaginary, due to the harmonic oscillator states being alternately even and odd. In Eq. (10), only the last term on the right-hand side contributes initially. Then, by choosing the initial overall phase of the leftpropagating end-fire mode to be the same as that of the right-propagating one, the left and right contributions for all odd trap levels cancel out since the overlap functions for those modes are purely imaginary. The cancellation is no longer exact if either the phases of the left and rightpropagating end-fire modes are not the same or if their initial amplitudes are not equal, as is in general the case due to fluctuations.

As the temperature approaches $T_{c}, \xi\left(\tau_{d}\right)$ undergoes a rapid initial decay on a timescale $\tau_{\text {th }}$ roughly an order of magnitude faster than the slow decay characterized by $\tau_{c}$. This is illustrated for $n_{c}=0.1$ as the solid line in Fig. [3(b). These two time scales were experimentally observed by Yoshikawa et al., see Fig. 3 in Ref. [17]. The appearance of a slow decay below the critical temperature $T_{c}$, combined with the disappearance of the rapid decay at $T \rightarrow 0$, clearly points to the fact that they are associated with the condensed and thermal fractions respectively. The existence of these two time scales was exploited by Sadler et al. [4] to image selectively the condensed fraction of an ultra-cold Bose gas below the critical temperature while remaining blind to the thermal fraction, as we discuss next. 


\section{THERMALLY SELECTIVE SCATTERING}

This section further discusses how the Doppler broadening of the thermal fraction inhibits superradiance when the effective Rabi frequency $\Omega_{R}$ becomes comparable to the trap frequency, thereby making superradiance a sensitive probe of the condensed fraction of the sample [4].

The circles in Fig. 4 (a) show the ratio $n_{\mathrm{th}}^{(2)} / n_{c}^{(2)}$, the number of atoms transferred to the electronic state $|2\rangle$ from the excited trap levels to the number of atoms transferred from the trap ground state to $|2\rangle$, at peak end-fire mode intensity and as a function of $\omega_{t} / \Omega_{R}$ for an initial condensed fraction $n_{c}=1 / 2$. As the ratio $\omega_{t} / \Omega_{R}$ increases, fewer thermal atoms participate in the superradiant scattering as a consequence of Doppler dephasing. The triangles, which show the total number of scattered atoms, confirm that indeed, the bulk of the superradiant emission is associated to the condensed atoms in that case.

This behavior is further illustrated by plotting the fraction of atoms left in the electronic state $|1\rangle$ at the end of the superradiant emission as a function of trap level. This is illustrated in Fig. 4(b) for the cases (from lowest to highest curve) $\omega_{t} / \Omega_{R}=0.25,0.5$ and 0.75 and 1.0. When superradiance occurs on a time scale faster than the inverse trap frequency, all levels contribute roughly in proportion to their initial occupation [26]. But as $\omega_{t} / \Omega_{R}$ becomes of order unity, the lower trap levels contribute significantly more in proportion to their initial occupation than the higher levels which Doppler dephase faster.

The selective scattering of atoms initially in the deepest trap levels implies that the scattered atoms are in effect colder than the unscattered atoms - a situation somewhat akin to evaporative cooling, except that in the present case it is the warmer atoms that are "left behind." The squares in Fig. 4(c) show the atomic distribution of atoms in electronic state $|2\rangle$ at the end of the superradiant emission for the first 20 trap levels for $n_{c}=0.5$, $\omega_{t} / \Omega_{R}=1.0$ and $N=10^{6}$. The solid circles show the initial distribution of thermal atoms in state $|1\rangle$ (the occupation number of the lowest trap level is off scale and is not shown here and the initial distribution was multiplied by a factor of 10 for visibility).

We use the root-mean-square deviation of the centerof-mass energy of the atoms as a measure of their temperature, and compare its initial value $\Delta \epsilon_{1}$ for atoms in the state $|1\rangle$, to that of the atoms in the electronic ground state $|2\rangle, \Delta \epsilon_{2}$, at the end of the superradiant emission. For $\omega_{t} / \Omega_{R}=1.0$ we find $\Delta \epsilon_{2}=\Delta \epsilon_{1} / 2$, a modest reduction in rms energy. This is despite the fact that several trap levels wind up being significantly populated, as opposed to only the lowest trap level being significantly populated initially and follows from the comparatively few thermal atoms undergoing Raman scattering, as we have seen. For the case at hand, $58 \%$ of the particles in the state $|1\rangle$ initially occupy the first 10 trap levels, but after scattering $82 \%$ of the particles scattered to $|2\rangle$ occupy these 10 levels.
This effect can be enhanced if the trap frequency is chosen so as to approximately match the photon recoil frequency, $\hbar q^{2} / 2 M \approx \omega_{t}$, the spatial width of the ground state of the atom trap thus being of the same order as the wavelength of light. This implies that the Lamb-Dicke parameter, $\eta=k_{L} \sqrt{\hbar / 2 m \omega_{t}}$, which serves as a measure of the amount of coupling between the motional and internal states of the atoms, is of order $\eta \simeq 1$. The overlap function associated with the ground state, $\eta_{0 m}(\mathbf{q})$, is then appreciable only for $m=1$, and the condensed atoms will only recoil to that level, instead of several levels as was the case in Fig. 4(c).

\section{CONCLUSION}

In this paper we have explored theoretically various aspects of superradiance from ultracold, but finite temperature atomic gases. In particular we've illustrated explicitly the existence of two well separated coherence timescales associated with the condensed and thermal fractions of the gas respectively. In the regime where Doppler dephasing plays an important role, we found that superradiant scattering takes place predominantly from atoms in the lowest lying trap levels as these are less prone to the Doppler dephasing. That effect was exploited in [4] to image selectively the condensed fraction of an ultra-cold gas. We demonstrated that as a consequence atoms in the scattered state have a reduced rms deviation from the average trap energy as compared to the atoms in the initial state, and are in that sense colder than atoms in the initial state.

Our calculations considered a system with end-fire modes recoiling perpendicularly to the pump beam. In a system where the pump beam propagates collinearly to the scattered beams, such as an elongated condensate pumped along the long axis, it is possible to suppress Raman scattering into the backward end-fire mode, as compared to the forward end-fire mode, by exploiting the same dephasing effects that lead to thermally selective scattering. This results because the recoil momentum associated with the forward end-fire mode is small compared to that due to backward scattering. Hence it is possible to realize conditions such that the forward scattering Lamb-Dicke parameter $\eta_{f} \ll 1$ and the backward scattering one $\eta_{b} \gg 1$, implying negligible coupling between the ground state and higher motional states for forward scattering, but strong coupling and accompanying dephasing for the backward scattering case. This effect would not be present in Rayleigh scattering experiments using the same geometry, since there the pump beam simply sees a phase shift in the forward direction and only the backward mode is superradiantly enhanced. 


\section{Acknowledgments}

It is a pleasure to thank Mishkat Battacharya, Wenzhou Chen, Omjyoti Dutta and Swati Singh for fruitful discussions.

This work is supported in part by the US Army Research Office, the National Science Foundation and the US Office of Naval Research.
[1] M. Greiner, O. Mandel, T. Esslinger, T. Hänsch, and I. Bloch, Nature 425, 39 (2002).

[2] S. Ritter, A. Öttl, T. Donner, T. Bourdel, M. Köhl, and T. Esslinger, Phys. Rev. Lett. 98, 090402 (2007).

[3] T. Donner, S. Ritter, T. Bourdel, A. Öttl, M. Köhl, and T. Esslinger, Science 315, 1556 (2007).

[4] L. E. Sadler, J. M. Higbie, S. R. Leslie, M. Vengalattore, and D. M. Stamper-Kurn, Phys. Rev. Lett. 98, 110401 (2007).

[5] N. Skribanowitz, I. P. Herman, J. C. MacGillivray, and M. S. Feld, Phys. Rev. Lett. 30, 309 (1973).

[6] M. Gross, C. Fabre, P. Pillet, and S. Haroche, Phys. Rev. Lett. 36, 1035 (1976).

[7] H. M. Gibbs, Q. H. F. Vrehen, and H. M. J. Hikspoors, Phys. Rev. Lett. 39, 547 (1977).

[8] A. T. Rosenberger and T. A. DeTemple, Phys. Rev. A 24, 868 (1981).

[9] R. Bonifacio and L. Lugiato, Phys. Rev. A 11, 1507 (1975).

[10] S. Inouye, A. P. Chikkatur, D. M. Stamper-Kurn, J. Stenger, D. E. Pritchard, and W. Ketterle, Science 285, 571 (1999).

[11] S. Inouye, T. Pfau, S. Gupta, A. P. Chikkatur, A. Görlitz, D. E. Pritchard, and W. Ketterle, Nature 402, 641 (1999).

[12] M. Kozuma, Y. Suzuki, Y. Torii, T. Sugiura, T. Kuga, E. W. Hagley, and L. Deng, Science 286, 2309 (1999).

[13] D. Schneble, G. K. Campbell, E. W. Streed, M. Boyd, D. E. Pritchard, and W. Ketterle, Phys. Rev. A 69, 041601(R) (2004).

[14] R. Bonifacio, G. R. M. Robb, and B. W. J. McNeil, Phys. Rev. A 56, 912 (1997).
[15] S. Slama, S. Bux, G. Krenz, C. Zimmermann, and P. W. Courteille, Phys. Rev. Lett. 98, 053603 (2007).

[16] S. Slama, G. Krenz, S. Bux, C. Zimmermann, and P. W. Courteille, Phys. Rev. A 75, 063620 (2007).

[17] Y. Yoshikawa, Y. Torii, and T. Kuga, Phys. Rev. Lett. 94, 083602 (2005).

[18] H. Uys and P. Meystre, Phys. Rev. A 75, 033805 (2007).

[19] M. G. Moore and P. Meystre, Phys. Rev. Lett. 83, 5202 (1999).

[20] M. Gronchi, L. A. Lugiato, and P. Butera, Phys. Rev. A 18, 689 (1978).

[21] F. Haake, H. King, G. Schröder, J. Haus, and R. Glauber, Phys. Rev. A 20, 2047 (1979).

[22] L. Pitaevskii and S. Stringari, Bose-Einstein Condensation (Oxford Science Publications, 2003).

[23] O. Zobay and G. M. Nikolopoulos, Phys. Rev. A 72, 041604(R) (2005).

[24] O. Zobay and G. M. Nikolopoulos, Phys. Rev. A 73, 013620 (2006).

[25] The relatively high trap frequency that we consider is guided solely by numerical considerations: an atom in the lowest trap level that experiences a recoil $\hbar k_{L}=2 \pi \hbar / 795$ $\mathrm{nm}$ along either of the axes is excited to a trap level $n_{r} \approx \hbar k^{2} / 2 M \omega_{t}$, or $n_{r} \approx 10^{3}$ for typical trap frequencies $\left(\omega_{t}=10 \pi \mathrm{rad} / \mathrm{s}\right.$ in [4] $)$. Our choice of $\omega_{t}$ reduces $n_{r}$ by two orders of magnitude, rendering the problem computationally significantly more tractable.

[26] The rapid rise at the tail-end of that curve is a consequence of the finite number of trap levels in the simulations. Since the population of high levels remains low at all times this artifact does not significantly affect our conclusions. 